\title{
Bone lesions in early syphilis detected by bone scintigraphy
}

\author{
KLAUS HANSEN, * KELD HVID-JACOBSEN, † HELLE LINDEWALD, \\ PER SOELBERG SøRENSEN,* AND KAARE WEISMANN§ \\ From the Departments of *Neurology, †Nuclear Medicine, $¥ N e u r o r a d i o l o g y$, and $\S D e r m a t o l o g y$ and \\ Venereology, Rigshospitalet, University of Copenhagen, Denmark
}

SUMMARY We report a case of early syphilis with multiple bone lesions which all resolved after treatment with penicillin. We discuss why bone lesions may be more prevalent than generally believed and why $99 \mathrm{~m}$-Tc-MDP-bone scintigraphy is more sensitive than radiography in detecting syphilitic periostitis.

\section{Introduction}

Destructive bone lesions in late and congenital syphilis are well known but have rarely been seen since the introduction of penicillin treatment. Bone lesions in early syphilis, however, are rarely recognised but are of considerable clinical interest as they may be the first or only evidence of syphilitic infection. Because they may be asymptomatic, can cause only slight pain which doctors and patients may neglect, or may be painful but (in the early phase) radiologically silent, incidence of such lesions can be underestimated.

We present a case of early syphilis in which painful osteoperiostitis was the major manifestation of the disease. Bone scintigraphy proved to be a sensitive diagnostic tool.

\section{Case report}

In March 1981 a 31 year old black man from the Ivory Coast was admitted to the department of neurology at this hospital with a seven month history of frontal headache and slight fever. While he was still in Africa in June 1980 he had experienced intense pains in both femora, which had disappeared spontaneously after a few months. Shortly after his arrival in Denmark in August 1980 he had developed a predominantly rightsided frontal headache and pain in the left lower jaw. He had previously experienced several episodes of urethral discharge, and in $\mathbf{1 9 7 6}$ he had a penile ulcer and swelling of the inguinal lymph nodes. At that time he had been

Address for reprints: Dr Klaus Hansen, Department of Neurology, Rigshospitalet, Blegdamsvej 9, 2100 Copenhagen $\varnothing$, Denmark

Accepted for publication 11 November 1983 treated with injections and tablets of unidentified drugs. In May 1980 he had developed another penile ulcer, which healed spontaneously. There was no history of head trauma, recent or past major infectious diseases, pulmonary symptoms, or skin rash. He had had no homosexual contact.

Examination disclosed severe tenderness over the right frontal bone and a round $10 \mathrm{~mm}$ scar on the shaft of the penis. There were no other skin changes, condylomata lata, peripheral lymphadenopathy, or abnormal cardiovascular or neurological findings.

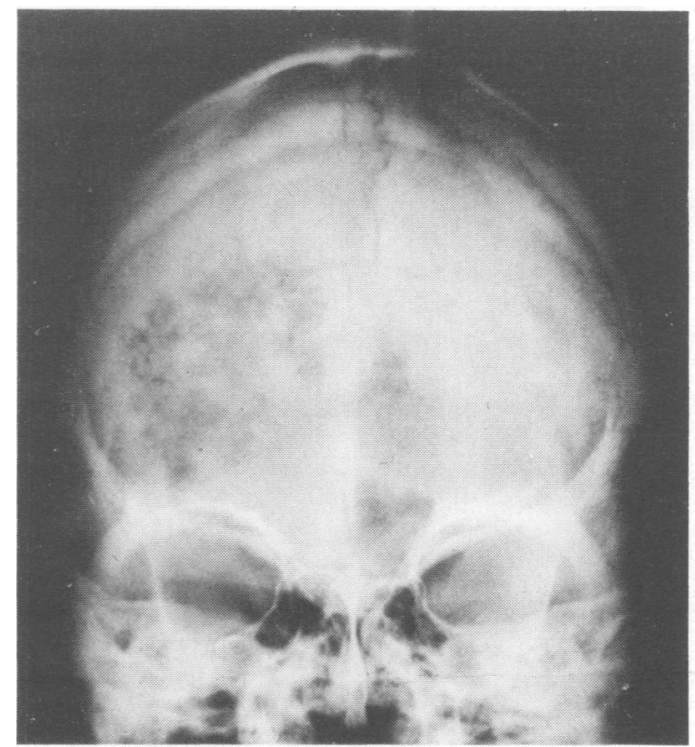

FIG $1 X$ ray film of the skull showing large area of bone destruction with porous and wormeaten appearance in the right side of the frontal bone extending over the midline. 
His temperature was $38^{\circ} \mathrm{C}$. Routine laboratory investigations were normal except for an erythrocyte sedimentation rate of $64 \mathrm{~mm}$ in the first hour and serum alkaline phosphatase activity of 340 IU/1 (normal activity is less than 275 IU/1). Fluorescent treponemal antibody-absorbed (FTA-ABS) and Treponema pallidum immobilisation (TPI) serological tests gave pusitive results, and there was a strong cardiolipin reaction (Wassermann). The cerebrospinal fluid was normal. A skull $x$ ray film showed a $6 \mathrm{~cm}$ osteolytic lesion in the right frontal bone (fig 1). Bone scintigraphy and additional $x$ ray examinations including computed tomography of the skull showed widespread bone lesions. Bone scintigraphy showed increased radionuclide uptake in the frontal lesion and several smaller lesions in the parietal, occipital, maxillary, and mandibular bones. Pathological radionuclide uptake was also seen in two ribs, the right inferior pubic ramus, the right

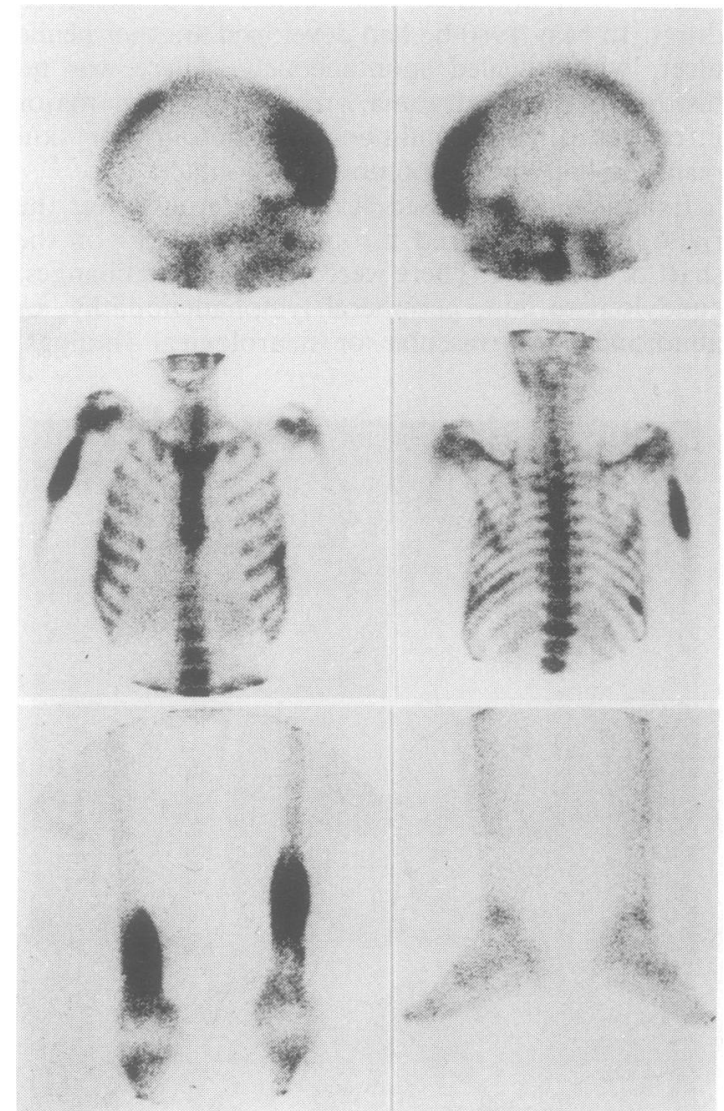

FIG $299 m-I c-M D P$-bone scintigraph showing multiple areas of pathological radionuclide uptake in the skull, ribs, and extremities. Note the periosteal-cortical involvement of both femora. humerus, and distally in both femora. The femoral lesions suggested that only the periosteum and cortex were affected, and not the bone marrow (fig 2).

Skeletal $x$ ray films showed destructive lesions in the left angle of the mandible and the right inferior pubic ramus, a destructive lesion with a periosteal reaction in the right humerus (fig 3), and isolated periostitis with cortical bone proliferation in both femora (fig 4). Bone scintigraphy showed several radiologically silent lesions, presumably representing an early phase of periostitis. A chest $x$ ray film showed a $5 \mathrm{~cm}$ round infiltrate in the posterior lower lobe of the left lung, but no changes in the ribs or sternum. The lung infiltrate was noted retrospectively on a $70 \times 70 \mathrm{~mm}$ chest $x$ ray film taken for routine tuberculosis control when the patient entered Denmark eight months earlier. An open bone biopsy specimen from the right frontal bone showed no specific changes.

Osteitis in early syphilis was diagnosed. He was treated with benzathine penicillin $\mathrm{G} \quad 2.4 \mathrm{MU}$ intramuscularly once a week for three successive weeks followed by two additional courses at monthly

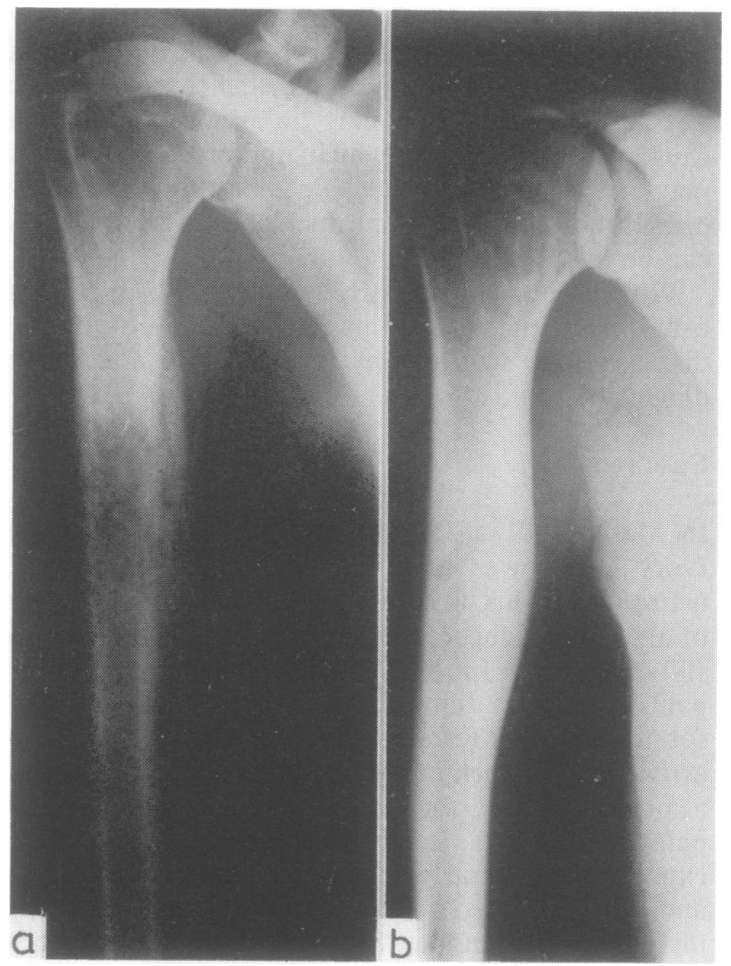

FIG $3 X$ ray film of the right humerus showing: a) large osteolytic lesion and irregular periosteal proliferation in the proximal shaft and, b) noticeable improvement with no destructive lesion five months after treatment. 


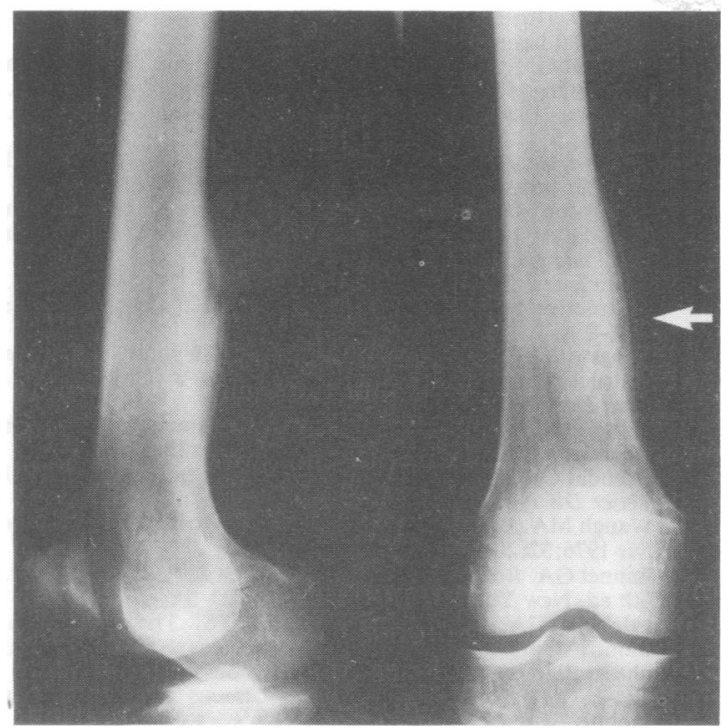

FIG 4 Anteroposterior and lateral projection of the right femur on $x$ ray films showing proliferative periostitis with periosteal elevation (arrow) and cortical bone proliferation in the distal shaft, but no involvement of the bone marrow.

intervals (a total of $21.6 \mathrm{MU}$ over five months), and had no Jarisch-Herxheimer reaction. Two days after the first injection the headache had diminished noticeably, and it disappeared completely within 14 days. His erythrocyte sedimentation rate and serum alkaline phosphatase activity returned to normal within three weeks. $X$ ray films taken five months later showed appreciable resolution of the lesions in the affected bones. Repeat bone scintigraphy showed no change after one month, but about a $50 \%$ reduction in activity after nine months, at which time the Wassermann reaction had become less strongly positive, whereas the FTA-ABS and TPI results remained unchanged. The lung infiltrate also resolved during penicillin treatment.

\section{Discussion}

The positive results to serological tests for syphilis and the rapid response to penicillin confirmed the diagnosis of early syphilis in our patient. The precise time of the primary infection cannot be ascertained, but may have been when he had insufficient treatment for a chancre in 1976 or a reinfection in 1980. A diagnosis of yaws was ruled out as he had lived in urban areas in Africa and had no cutaneous signs of this condition. ${ }^{3}$

The incidence of bone lesions in early syphilis is not known, but estimates have ranged from $0.15 \%$ to $36 \% .{ }^{14}$ These figures were based on clinical observations without radiological examination, and the material was not collected from a consecutive series of patients. In another study routine skull $x$ ray examination was performed on 80 consecutive patients with secondary syphilis. ${ }^{5}$ Cranial lesions were seen in seven $(8 \%)$, three of whom did not complain of bone pain. As asymptomatic bone lesions could have been present and total skeletal $x$ ray examinations were not performed, the authors estimated that the true incidence of bone lesions was higher than $\mathbf{8 \%}$. Bone lesions in early syphilis may also be overlooked because they are radiologically invisible in the early phase or because headache due to a mild aseptic meningitis is a common symptom (occurring in about $15 \%$ of all cases) of secondary syphilis. ${ }^{3}$

During the past decade a few cases of early syphilis with destructive bone lesions have been reported. ${ }^{26-9}$ Some reports have found bone lesions to be the first manifestation of the diseases. ${ }^{2}{ }^{2}$ Bone pains have been reported in patients with early syphilis but normal roentgenograms, ${ }^{1}{ }^{10}$ and in some reports radiologically visible changes only developed several months after the initial pain. 9 Syphilitic bone disease is probably initially generalised proliferative periostitis due to vasculitis, occasionally followed by a focal osteitis and eventually osteomyelitis. ${ }^{11}$ This sequence may explain the late appearance of radiological findings. Superficial bones are most frequently affected, primarily those of the skull, the sternoclavicular region, and the long bones of the legs, especially the tibiae. ${ }^{11}$ The skull lesions are often prelambdoidal ${ }^{15}$ and mainly affect the tabula externa. ${ }^{1}$ They are irregular osteolytic areas with a wormeaten appearance. The typical picture in long bones is initially a periosteal proliferative reaction with periosteal elevation followed by deeper destructive and proliferative changes causing swelling, osteolysis, and formation of sequestra. The bone scintigram of the femoral lesions of our patient showed that the bone marrow was spared, which indicated mainly periosteal-cortical involvement. This accords with proliferative periostitis which is the major osseous manifestation of early skeletal syphilis, and which in this case suggested syphilitic infection. It has been stated that early and late syphilitic lesions can be differentiated radiologically, ${ }^{5}$ but this seems to be uncertain. ${ }^{6}$

Few authors have succeeded in isolating spirochaetes from bone lesions in early syphilis. ${ }^{8}$ Such investigation therefore only seems to be indicated if there is no response to antisyphilitic treatment or the diagnosis is in doubt.

Bone scintigraphy seems to be more sensitive than radiography in detecting skeletal lesions in early 
syphilis, especially at the stage of periostitis. Even minor foci of increased mineral turnover will accumulate radionuclide, whereas about $50 \%$ decalcification is necessary to make bone lesions visible on radiography. ${ }^{12}$ In our patient as well as in the only previously reported case in which bone scintigraphy was performed, ${ }^{2}$ this investigation showed all lesions visible on radiography and several other clinically and radiologically silent lesions. The higher sensitivity of scintigraphy explains why resolution was seen later using this examination than using radiography. The lesions seen on $x$ ray films showed noticeable improvement within a few months after treatment, as in an earlier study in which about 10 months were needed for complete healing. ${ }^{13} \mathrm{We}$ recommend bone scintigraphy as a sensitive diagnostic tool in early skeletal syphilis, whereas radiography is preferable for follow up of the response to treatment.

\section{References}

1. Reynolds FW, Wasserman H. Destructive osseous lesions in early syphilis. Arch Intern Med 1942;69:263-76.
2. Siegel D, Hirschman SZ. Syphilitic osteomyelitis with diffusely abnormal bonescan. Mt Sinai J Med (NY) 1979; 46:320-3.

3. Lomholt G. Syphilis, yaws and pinta. In: Rook A, Wilkinson DS, Ebling FJG, eds. Textbook of dermatology, 3rd ed. London: Blackwell Scientific Publications, 1976;701-47. (Vol 1).

4. Wile VJ, Senear FE. A study of the involvement of bone and joints in early syphilis. Am J Med Sci 1976; 152:689-93.

5. Thompson CRG, Priston RH. Lesions of the skull in secondary syphilis. American Journal of Syphilis, Gonorrhea and Venereal Disease 1952;36:332-41.

6. Dismukes VE, Delgade DG, Mallernee SV Myers TC. Destructive bone disease in early syphilis. $J A M A$ 1976; 236: 2646-8.

7. Longstrith $\mathrm{P}$, Hoke AW, McElroy C. Hepatitis and bone destruction as uncommon manifestations of early syphilis. Arch Dermatol 1976;112:1451-4.

8. Shore RN, Kiesel HA, Bennett HD. Osteolytic lesions in secondary syphilis. Arch Intern Med 1977; 137:1465-7.

9. Graudal $\mathrm{C}$, Lange $\mathrm{K}$, Jensen $\mathrm{O}$. Osteitis in early syphilis. $\mathrm{Br} J$ Vener Dis 1981;57:312-4.

10. Waugh MA. Bony symptoms in secondary syphilis. Br J Vener Dis 1976; 52: 204-5.

11. Bennet GA. Bones. In: Anderson WAD, ed. Pathology. Vol 2. 6th ed. New York: CV Mosby, 1971;1707-10.

12. Edelstyn GA, Gillespit PJ, Grebbel FS. The radiological demonstration of osseus metastases. Experimental observations. Clin Radiol 1967; 18: 158-62.

13. Glaser RJ, Scott V. Destructive osseous lesions in early syphilis. Am J Med 1947; 3:496-500. 